\title{
CUSP FORMS ASSOCIATED TO RANK 2 PARABOLIC SUBGROUPS OF KLEINIAN GROUPS
}

\author{
IRWIN KRA
}

(Communicated by Clifford J. Earle, Jr.)

\begin{abstract}
To each rank 2 parabolic subgroup of a Kleinian group $\Gamma$, we associate a cusp form and a linear functional on the Eichler cohomology space $P H^{1}\left(\Gamma, \Pi_{2 q-2}\right)$. We explore the relation between these two objects and as a consequence we evaluate certain Poincaré series for rank 2 parabolic groups.
\end{abstract}

This paper is a continuation of the study of automorphic forms associated to elements of Kleinian groups. We treated the case of cusp forms associated to loxodromic elements in [K3] and the holomorphic forms associated to parabolic elements in [K1]. We combine ideas from [K3] and [K1] to investigate the cusp forms associated to rank 2 parabolic subgroups of Kleinian groups.

Let $A$ and $B$ be generators of a rank 2 parabolic subgroup $G$ of a Kleinian group $\Gamma$. We associate (in $\S 2)$ to the pair $(A, B)$ a linear functional

$$
l_{A, B}: P H^{1}\left(\Gamma, \Pi_{2 q-2}\right) \rightarrow \mathbb{C}
$$

on the space of parabolic cohomology classes for $\Gamma,{ }^{1}$ and (in $\S 3$ ) a cusp form $\varphi_{A, B} \in \mathbb{A}_{q}(\Omega, \Gamma)$. One of the main results is (Theorem 4.3) the connection between these two invariants provided by the Bers embedding

$$
\beta^{*}: \mathbb{A}_{q}(\Omega, \Gamma) \rightarrow P H^{1}\left(\Gamma, \Pi_{2 q-2}\right)
$$

and the Petersson scalar product

$$
\langle\cdot, \cdot\rangle: \mathbb{A}_{q}(\Omega, \Gamma) \times \mathbb{A}_{q}(\Omega, \Gamma) \rightarrow \mathbb{C} .
$$

A by-product of this work is the evaluation of some naturally occurring elliptic sums. See $\S 5$.

\section{INVARIANTS FOR PARABOLIC ELEMENTS}

1.1. A parabolic Möbius transformation $A \in P S L(2, \mathbb{C})$ has a unique fixed point $\alpha=\alpha(A) \in \hat{\mathbb{C}}=\mathbb{C} \cup\{\infty\}$ and a well-defined translation length

Received by the editors October 30, 1989.

1980 Mathematics Subject Classification (1985 Revision). Primary 30F40, 32N10, $20 \mathrm{H} 10$.

Research partially supported by National Science Foundation grants DMS 8701774 and DMS 8505550

${ }^{1}$ We will use the notation and terminology of [K2], [K1] and [K3]. 
$\tau=\tau(A) \in \mathbb{C}^{*}=\mathbb{C}-\{0\}$. In normal form:

$$
A(z)=z+\tau, \quad A=\left[\begin{array}{ll}
1 & \tau \\
0 & 1
\end{array}\right] \quad \text { if } \alpha=\infty,
$$

and

$$
\frac{1}{A(z)-\alpha}=\frac{1}{z-\alpha}+\tau, \quad A=\left[\begin{array}{cc}
1+\alpha \tau & -\alpha^{2} \tau \\
\tau & 1-\alpha \tau
\end{array}\right] \quad \text { if } \alpha \in \mathbb{C} .
$$

For future use, we observe that for finite $\alpha$,

$$
A^{\prime}(\alpha)=1 \text { and } A^{\prime \prime}(\alpha)=-2 \tau .
$$

If $C$ is an arbitrary Möbius transformation, then

$$
\alpha\left(C \circ A \circ C^{-1}\right)=C(\alpha(A)) \text { and } \tau\left(C \circ A \circ C^{-1}\right)=\tau(A) / C^{\prime}(\alpha) .
$$

The above formulae must be properly interpreted for $\alpha$ or $C(\alpha)=\infty$. Assume, for example, that $\alpha \in \mathbb{C}$ and $C(\alpha)=\infty$. Write

$$
C=\left[\begin{array}{cc}
\beta & \delta \\
1 & -\alpha
\end{array}\right] \in P G L(2, \mathbb{C}) \text {. }
$$

Then $C^{\prime}(z)=-(\alpha \beta+\delta) /\left((z-\alpha)^{2}\right)$ and we must interpret $C^{\prime}(\alpha)$ as $1 /(\alpha \beta+\delta)$.

1.2. The holomorphic differential form on $\hat{\mathbb{C}}-\alpha$ for the cyclic group $\langle A\rangle$ is defined by

$$
h(z)=h_{A}(z)=\frac{1}{\tau(A)} \quad \text { if } \alpha=\infty,
$$

and

$$
h(z)=h_{A}(z)=\frac{1}{\tau(A)} \frac{-1}{(z-\alpha)^{2}} \quad \text { if } \alpha \in \mathbb{C} .
$$

The function $h$ is canonically associated to the Möbius transformation $A$ and is characterized by the properties

$$
\int_{z_{0}}^{A\left(z_{0}\right)} h(z) d z=1, \quad \text { all } z_{0} \in \hat{\mathbb{C}}-\{\alpha\}
$$

(as long as the path of integration stays in $\hat{\mathbb{C}}-\{\alpha\}$ ), and $h(z) d z$ has simple poles at the two punctures of $(\hat{\mathbb{C}}-\{\alpha\}) /\langle A\rangle$.

As a consequence of $(1.1 .1)$ and the basic identity ${ }^{2}$

$$
C(z)-C(\zeta)=(z-\zeta) C^{\prime}(z)^{1 / 2} C^{\prime}(\zeta)^{1 / 2},
$$

which is valid for all $C \in \operatorname{PSL}(2, \mathbb{C})$ and all $z$ and $\zeta \in \hat{\mathbb{C}}$, we have

$$
h_{C \circ A \circ C^{-1}}(C(z)) C^{\prime}(z)=h_{A}(z), \quad \text { all } z \in \hat{\mathbb{C}},
$$

or equivalently,

$$
C_{q}^{*}\left(h_{C \circ A \circ C^{-1}}^{q}\right)=h_{A}^{q}, \quad \text { all } q \in \mathbb{Z} .
$$

\footnotetext{
${ }^{2}$ This formula (and similar formulae) must be adjusted if $z, \zeta, C(z)$, or $C(\zeta)$ equals $\infty$. We leave such modifications to the reader (generally).
} 
1.3. The polynomial ring of $A$ acts on the right on the vector space $\Pi_{2 q-2}$ of polynomials of degree $\leq 2 q-2$ by the Eichler action (from now on $q \geq 2$ ). The kernel of the operator [ $A-I]$ is the one-dimensional space with basis $h_{A}^{1-q}$. The image of $[A-I]$ consists of

$$
\Pi_{2 q-2}(\alpha)=\left\{v \in \Pi_{2 q-2} ; v(\alpha)=0\right\} .
$$

For details see $[\mathrm{K} 2, \S 8.3]^{3}$

\section{LINEAR FUNCTIONALS ON $P H^{1}\left(\Gamma, \Pi_{2 q-2}\right)$ ASSOCIATED TO RANK 2 PARABOLIC SUBGROUPS OF $\Gamma$}

2.1. Let $(A, B)$ be an ordered pair of generators for a rank 2 parabolic subgroup $G$ of a Kleinian group $\Gamma$. Let $\chi_{1}$ be a cocycle representing a cohomology class in $P H^{1}\left(\Gamma, \Pi_{2 q-2}\right)$. Choose $v \in \Pi_{2 q-2}$ such that

$$
\chi_{1}(A)=v \cdot A-v \text {. }
$$

Then the cocycle

$$
\chi(\gamma)=\chi_{1}(\gamma)-v \cdot \gamma+v, \quad \gamma \in \Gamma,
$$

is cohomologous to $\chi_{1}$ and satisfies

$$
\chi(A)=0 .
$$

The cocycle relation

$$
\chi(A) \cdot B+\chi(B)=\chi(A \circ B)=\chi(B \circ A)=\chi(B) \cdot A+\chi(A)
$$

is a consequence of the fact that $A$ and $B$ commute. It implies that $\chi(B) \in$ kernel $[A-I]$. Thus there exists a $l(\chi) \in \mathbb{C}$ such that

$$
\chi(B)=l(\chi) h_{A}^{1-q} \text {. }
$$

Proposition. (a) The rule

$$
\chi \longmapsto l(\chi)=l_{A, B}(\chi)
$$

defines an element of the dual space $P H^{1}\left(\Gamma, \Pi_{2 q-2}\right)^{*}$ of $P H^{1}\left(\Gamma, \Pi_{2 q-2}\right)$.

(b) For all $C \in \Gamma$,

$$
l_{C \circ A \circ C^{-1}, C \circ B \circ C^{-1}}=l_{A, B} \text {. }
$$

Proof. Let $\chi_{1}$ be an arbitrary cocycle with $\chi_{1}(A)=0$ and $\chi_{1}$ cohomologous to $\chi$. Then there exists a $v \in \Pi_{2 q-2}$ such that

$$
\chi_{1}(\gamma)=\chi(\gamma)-v \cdot \gamma+v, \quad \gamma \in \Gamma .
$$

It follows that $v \in \operatorname{kernel}[A-I]=\operatorname{kernel}[B-I]$. Thus $\chi_{1}(B)=\chi(B)$ and $l$ is well defined.

2.2. For arbitrary $C \in P S L(2, \mathbb{C})$, the linear map

$$
\wedge: P H^{1}\left(\Gamma, \Pi_{2 q-2}\right) \rightarrow P H^{1}\left(C \Gamma C^{-1}, \Pi_{2 q-2}\right)
$$

\footnotetext{
${ }^{3}$ There are a number of misprints in [K2]. For example, the formula for Image $(E-1)$ in $\S 8.3$ should read Image $(E-1)=\left\{p \in \Pi_{2 q-2} ; p(a)=0\right\}$.
} 
defined on the cocycle level by

$$
\hat{\chi}\left(C \circ \gamma \circ C^{-1}\right)=\chi(\gamma) \cdot C^{-1}, \quad \gamma \in \Gamma,
$$

is a (surjective) isomorphism. For $\chi \in P Z^{1}\left(\Gamma, \Pi_{2 q-2}\right)$ with

$$
\chi(A)=0 \quad \text { and } \quad \chi(B)=l(\chi) h_{A}^{1-q}
$$

we have

$$
\hat{\chi}\left(C \circ A \circ C^{-1}\right)=0 \quad \text { and } \quad \hat{\chi}\left(C \circ B \circ C^{-1}\right)=l(\chi) h_{C \circ A \circ C^{-1}}^{1-q} ;
$$

which shows that

$$
l_{A, B}(\chi)=l_{C \circ A \circ C^{-1}, C \circ B \circ C^{-1}}(\hat{\chi}) .
$$

2.3. If $C \in \Gamma$, then for every cocycle $\chi$ for $\Gamma$,

$$
\chi\left(C \circ \gamma \circ C^{-1}\right)=\chi(\gamma) \cdot C^{-1}+\chi(C) \cdot C^{-1} \cdot\left[C \circ \gamma \circ C^{-1}-I\right]
$$

for all $\gamma \in \Gamma$. Thus $\chi$ is cohomologous to $\hat{\chi}$ and we conclude that

$$
l_{C \circ A \circ C^{-1}, C \circ B \circ C^{-1}}(\chi)=l_{C \circ A \circ C^{-1}, C \circ B \circ C^{-1}}(\hat{\chi})=l_{A, B}(\chi) .
$$

We have completed the proof of Proposition 2.1.

2.4. Let $\left(A_{1}, B_{1}\right)$ be a second ordered pair of generators for the group $G$. Then, as is well known, there exist integers $a, b, c, d$ so that

$$
A_{1}=A^{a} \circ B^{b}, \quad B_{1}=A^{c} \circ B^{d} \text { and } a d-b c=\varepsilon= \pm 1 .
$$

We are interested in comparing $l_{A_{1}, B_{1}}$ with $l_{A, B}$. As a consequence of (2.2.1), we may assume that $A=\left[\begin{array}{ll}1 & 1 \\ 0 & 1\end{array}\right]$ and $B=\left[\begin{array}{ll}1 & \tau \\ 0 & 1\end{array}\right]$ with $\operatorname{Im} \tau \neq 0$. Thus

$$
A_{1}=\left[\begin{array}{cc}
1 & a+b \tau \\
0 & 1
\end{array}\right], \quad B_{1}=\left[\begin{array}{cc}
1 & c+d \tau \\
0 & 1
\end{array}\right] .
$$

Let us choose $\chi \in P Z^{1}\left(\Gamma, \Pi_{2 q-2}\right)$ with

$$
\chi(A)=0 \quad \text { and } \quad \chi(B)=l(\chi) .
$$

Then

$$
\chi\left(A_{1}\right)=\chi\left(B^{b}\right)=\chi(B) \cdot\left[B^{b-1}+\cdots+I\right]=b l(\chi), \quad \chi\left(B_{1}\right)=d l(\chi) .
$$

We must replace the cocycle $\chi$ by a cohomologous cocycle that vanishes at $A_{1}$. Let

$$
v(z)=\frac{b l(\chi)}{a+b \tau} z, \quad z \in \mathbb{C} .
$$

The cocycle

$$
\chi_{1}(\gamma)=\chi(\gamma)-v \cdot \gamma+v, \quad \gamma \in \Gamma
$$

vanishes at $A_{1}$ and has value

$$
\chi_{1}\left(B_{1}\right)=\frac{\varepsilon}{a+b \tau} l(\chi)
$$


It follows that for all $\chi \in P H^{1}\left(\Gamma, \Pi_{2 q-2}\right)$,

$$
l_{A_{1}, B_{1}}(\chi)=\frac{\varepsilon}{(a+b \tau)^{q}} l_{A, B}(\chi) .
$$

Corollary. The projective class of the linear functional $l_{A, B} \in P H^{1}\left(\Gamma, \Pi_{2 q-2}\right)^{*}$ depends only on the conjugacy class of $G$ in $\Gamma$.

2.5. Let $\Delta$ denote the unit disk, and

$$
\Phi: \Delta \rightarrow \operatorname{Hom}^{\mathrm{par}}(\Gamma, P S L(2, \mathbb{C}))
$$

be a normalized holomorphic family of parabolic homomorphisms on $\Delta$; this means that for each $t \in \Delta, \Phi(t)$ is a homomorphism of the Kleinian group $\Gamma$ into $\operatorname{PSL}(2, \mathbb{C})$,

$$
\gamma(t)=\Phi(t)(\gamma)
$$

defines a holomorphic map from $\Delta$ to $\operatorname{PSL}(2, \mathbb{C})$ for all $\gamma \in \Gamma$ and is parabolic or the identity whenever $\gamma \in \Gamma$ is parabolic, and $\Phi(0)$ is the identity isomorphism. This normalized family $\Phi$ defines a cocycle $\chi$ for $q=2$ by

$$
\chi(\gamma)(z)=\dot{\gamma}(z) / \gamma^{\prime}(z), \quad z \in \mathbb{C},
$$

where

$$
\dot{\gamma}(z)=\left.\frac{\partial}{\partial t} \gamma(t)(z)\right|_{t=0}, \quad \gamma \in \Gamma, \quad z \in \mathbb{C} .
$$

For details, see [GK]. If there exists a holomorphic function $C$ from a nonempty neighborhood $\{t \in \mathbb{C} ;|t|<\varepsilon\}$ of 0 in $\mathbb{C}$ into $\operatorname{PSL}(2, \mathbb{C})$ with $C(0)$ $=I$ and

$$
\gamma(t)=C(t) \circ \gamma \circ C(t)^{-1}, \quad \text { all } \gamma \in \Gamma, \text { all }|t|<\varepsilon,
$$

then $\chi$ is a coboundary.

If $A$ and $B$ generate a rank 2 parabolic subgroup of $\Gamma$, then for sufficiently small $|t|$, we can define a holomorphic function by

$$
\tau(t)=\frac{\tau(B(t))}{\tau(A(t))} .
$$

Proposition. We have

$$
\tau^{\prime}(0)=l_{A, B}(\chi)
$$

Proof. By conjugation, we may assume that $A=\left[\begin{array}{ll}1 & 1 \\ 0 & 1\end{array}\right], B=\left[\begin{array}{ll}1 & \tau_{0} \\ 0 & 1\end{array}\right]$ with Im $\tau_{0} \neq 0$. Write

$$
A(t)=\left[\begin{array}{cc}
1+a(t) & b(t) \\
-\frac{a^{2}(t)}{b(t)} & 1-a(t)
\end{array}\right]
$$

with $a$ and $b$ holomorphic functions with $a(0)=0, b(0)=1$. It follows that

$$
\alpha(A(t))=-\frac{b(t)}{a(t)} \text {. }
$$

Since $\alpha(B(t))=\alpha(A(t))$, it also follows that

$$
B(t)=\left[\begin{array}{cc}
1+\beta(t) \frac{a(t)}{b(t)} & \beta(t) \\
-\frac{a^{2}(t) \beta(t)}{b^{2}(t)} & 1-\beta(t) \frac{a(t)}{b(t)}
\end{array}\right]
$$


with $\beta$ some holomorphic function satisfying $\beta(0)=\tau_{0}$. Let

$$
C(t)=\left[\begin{array}{cc}
b(t)^{-1 / 2} & 0 \\
a(t) b(t)^{-1 / 2} & b(t)^{1 / 2}
\end{array}\right]
$$

Then

and

$$
C(t) \circ A(t) \circ C(t)^{-1}=\left[\begin{array}{ll}
1 & 1 \\
0 & 1
\end{array}\right]
$$

$$
C(t) \circ B(t) \circ C(t)^{-1}=\left[\begin{array}{cc}
1 & \beta(t) / b(t) \\
0 & 1
\end{array}\right] .
$$

Since conjugation preserves the trace and does not change the cohomology class, it suffices to assume that

$$
A(t)=\left[\begin{array}{ll}
1 & 1 \\
0 & 1
\end{array}\right] \text { and } B(t)=\left[\begin{array}{cc}
1 & \tau(t) \\
0 & 1
\end{array}\right]
$$

with $\tau$ a holomorphic function in some neighborhood of 0 with $\tau(0)=\tau_{0}$. It follows that $\chi(A)=0$ and $\chi(B)=\tau^{\prime}(0)=l_{A, B}(\chi)$.

3. CUSP FORMS ASSOCIATED TO RANK 2 PARABOLIC SUBGROUPS OF KLEINIAN GROUPS

3.1. Let $A$ and $B$ generate a rank 2 parabolic subgroup $G$ of the Kleinian group $\Gamma$. Then

$$
\varphi_{A, B}(z)=\sum_{\gamma \in G \backslash \Gamma} h_{A}(\gamma z)^{q} \gamma^{\prime}(z)^{q}, \quad z \in \Omega,
$$

defines a cusp form for $\Gamma$ (that is, an element of $\mathbb{A}_{q}(\Omega, \Gamma)$; here $\Omega$ is the region of discontinuity of $\Gamma$ ).

3.2. For arbitrary $C \in \Gamma$, we have

$$
\varphi_{C \circ A \circ C^{-1}, C \circ B \circ C^{-1}}=\varphi_{A, B}
$$

as a consequence of (1.2.3).

3.3. The cusp form $\varphi_{A, B}$ is related to a holomorphic Eichler integral. Assume that $\Gamma$ is a finitely generated Kleinian group. Then

$$
\psi_{A, B}(z)=\sum_{\gamma \in \Gamma / G} \frac{\gamma^{\prime}(\alpha)^{q}}{\gamma(\alpha)-z}, \quad z \in \Omega,
$$

(here $\alpha=\alpha(A)$ ) defines a holomorphic Eichler integral (that is, an element of $\left.\mathbb{E}_{1-q}^{c}(\Omega, \Gamma)\right)$, with

$$
\begin{aligned}
\frac{d^{2 q-1}}{d z^{2 q-1}} \psi_{A, B}(z) & =(2 q-1) ! \sum_{\gamma \in \Gamma / G} \frac{\gamma^{\prime}(\alpha)^{q}}{(\gamma(\alpha)-z)^{2 q}} \\
& =(2 q-1) ! \sum_{\gamma \in G \backslash \Gamma} \frac{\gamma^{\prime}(z)^{q}}{(\gamma(z)-\alpha)^{2 q}} \\
& =(-1)^{q} \tau(A)^{q}(2 q-1) ! \varphi_{A, B} .
\end{aligned}
$$


The Eichler integral $\psi$ is not parabolic with respect to $A$. It satisfies

$$
\begin{gathered}
(\operatorname{pd} \psi)(A)(\alpha)=\left(q-\frac{1}{2}\right) A^{\prime \prime}(\alpha)=(1-2 q) \tau(A), \\
(\operatorname{pd} \psi)(B)(\alpha)=(1-2 q) \tau(B) .
\end{gathered}
$$

The above formulae assume that $\langle A, B\rangle$ is the stabilizer of $\alpha$ in $\Gamma$. Adjustments need to be made when $\langle A, B\rangle$ is a proper subgroup of the stabilizer. The cohomology class pd $\psi$ is parabolic with respect to every parabolic element that fixes a point not $\Gamma$-equivalent to $\alpha$. For details, see [K1].

3.4. As a consequence of $\xi 3.3$, we see that $\varphi_{A, B} \neq 0$ provided $\alpha$ is accessible (defined in $[\mathrm{K} 1, \S 2]$ ) and $\langle A, B\rangle$ is the stabilizer of $\alpha$ in $\Gamma$. As a matter of fact, under this hypothesis, whenever $A$ (or $B$ ) fixes a component $\Delta$ of $\Omega$, then $\varphi_{A, B} \mid \Delta \neq 0$. More generally, let $\alpha$ be an accessible limit point of $\Gamma$ whose stabilizer $\Gamma(\alpha)$ contains a maximal rank 2 parabolic subgroup $P(\alpha)$ generated by $A$ and $B$ as above. Then (see [K1, $\S 0]) \Gamma(\alpha) / P(\alpha)$ is a cyclic group of order $n=1,2,3,4$ or 6 . The fixed point $\alpha$ is $q$-admissible if $q \equiv 0(\bmod$ $n)$. We showed in $[\mathrm{K} 1, \S 5]$ that $\varphi_{A, B} \neq 0$ if and only if $\alpha$ is $q$-admissible.

\section{LINEAR FUNCTIONALS ON CUSP FORMS ASSOCIATED TO RANK 2 PARABOLIC SUBGROUPS}

4.1. Let $\Gamma$ be a nonelementary Kleinian group. The Bers map

$$
\beta^{*}: \mathbb{A}_{q}(\Omega, \Gamma) \rightarrow P H^{1}\left(\Gamma, \Pi_{2 q-2}\right)
$$

is discussed in [K2, §0.2]. For $\psi \in \mathbb{A}_{q}(\Omega, \Gamma)$, we let $F$ be a potential for $\mu=\lambda^{2-2 q} \bar{\psi}$ (here $\lambda$ is the Poincare metric on the region of discontinuity $\Omega$ of $\Gamma$ ) and $\beta^{*}(\psi)=\operatorname{pd} F$. We need to construct specific potentials for $\mu$.

4.2. Let $A$ be a parabolic element of $\Gamma$. Let $F$ be a potential for $\mu$ that vanishes at $A^{j}(a), j=0,1, \ldots, 2 q-2$, where $a \in \hat{\mathbb{C}}$ is arbitrary with $a \neq \alpha=\alpha(A)$. We claim that $(\operatorname{pd} F)(A)=0$. The polynomial $(\operatorname{pd} F)(A)=$ $F \cdot A-F$ automatically vanishes at $\alpha$ and at $A^{j}(a), j=0, \ldots, 2 q-3$. It is thus identically zero. Thus we see that

$$
F(A(z)) A^{\prime}(z)^{1-q}-F(z)=0, \quad \text { all } z \in \hat{\mathbb{C}} .
$$

It follows that $F(\alpha)=0$ since by induction $F\left(A^{j}(a)\right)=0$ for all $j \in \mathbb{Z}$ and $\lim _{j \rightarrow \infty} A^{j}(a)=\alpha$ (the potential $F$ is a continuous function).

As a consequence, the potential $F_{1}$ for $\mu$ that vanishes at $\alpha$ and at $A^{j}(a)$, $j=0, \ldots, 2 q-3$, agrees with $F$. We have constructed two potentials

$$
F(z)=\frac{p(z)}{2 \pi i} \iint_{\Omega} \frac{\mu(\zeta)}{(\zeta-z) p(\zeta)} d \zeta \wedge d \bar{\zeta}, \quad z \in \hat{\mathbb{C}}
$$

that satisfy (4.2.1) by choosing

$$
p(z)=\prod_{j=0}^{2 q-2}\left(z-A^{j}(a)\right) \quad \text { or } \quad p(z)=(z-\alpha) \prod_{j=0}^{2 q-3}\left(z-A^{j}(a)\right) .
$$


4.3. We can now formulate the following

Theorem. Let $A$ and $B$ generate a rank 2 parabolic subgroup of the Kleinian group $\Gamma$. Then for all $\psi \in \mathbb{A}_{q}(\Omega, \Gamma)$,

$$
l_{A, B}\left(\beta^{*}(\psi)\right)=-i\left\langle\varphi_{A, B}, \psi\right\rangle .
$$

Proof. Let $a \in \hat{\mathbb{C}}, a \neq \alpha=\alpha(A)$. Let $F$ be the potential for $\mu=\lambda^{2-2 q} \bar{\psi}$ that vanishes at $A^{j}(a), j=0, \ldots, 2 q-2$. Then, as observed above, for $\chi=\operatorname{pd} F$, $l=l_{A, B}$, we have $\chi(A)=0$, and hence

$$
\chi(B)(z)=l(\chi)(-\tau(A))^{q-1}(z-\alpha)^{2 q-2}, \quad z \in \hat{\mathbb{C}} .
$$

Let $\omega$ be a standard fundamental domain for $\langle A, B\rangle$ such that $\partial \omega$ consists of oriented sides $c_{1}, c_{2},-A\left(c_{1}\right),-B\left(c_{2}\right)$ and the winding number of $\partial \omega$ around $\alpha$ is -1 . We compute

$$
\left(\frac{-1}{\tau(A)}\right)^{q} \int_{\partial \omega} \frac{F(z) d z}{(z-\alpha)^{2 q}}=\left(\frac{-1}{\tau(A)}\right)^{q}\left[\int_{c_{1}}-\int_{A\left(c_{1}\right)}+\int_{c_{2}}-\int_{B\left(c_{2}\right)}\right] \frac{F(z) d z}{(z-\alpha)^{2 q}} .
$$

Now

$$
\int_{c_{1}} \frac{F(z) d z}{(z-\alpha)^{2 q}}=\int_{c_{1}} \frac{F(A z) A^{\prime}(z)^{1-q} d z}{(A z-\alpha)^{2 q} A^{\prime}(z)^{-q}}=\int_{A\left(c_{1}\right)} \frac{F(z) d z}{(z-\alpha)^{2 q}},
$$

and

$$
\begin{aligned}
\int_{c_{2}} \frac{F(z) d z}{(z-\alpha)^{2 q}} & =\int_{c_{2}} \frac{F(B z) B^{\prime}(z)^{1-q}-\chi(B)(z)}{(z-\alpha)^{2 q}} d z \\
& =\int_{B\left(c_{2}\right)} \frac{F(z) d z}{(z-\alpha)^{2 q}}-l(\chi)(-\tau(A))^{q-1} \int_{c_{2}} \frac{d z}{(z-\alpha)^{2}} .
\end{aligned}
$$

We conclude that

$$
\left(\frac{-1}{\tau(A)}\right)^{q} \int_{\partial \omega} \frac{F(z) d z}{(z-\alpha)^{2 q}}=-l(\chi) .
$$

On the other hand $(G=\langle A, B\rangle)$

$$
\begin{aligned}
\left(\frac{-1}{\tau(A)}\right)^{q} \int_{\partial \omega} \frac{F(z) d z}{(z-\alpha)^{2 q}} & =-\left(\frac{-1}{\tau(A)}\right)^{q} \iint_{\omega} \frac{\partial}{\partial \bar{z}}\left[\frac{F(z)}{(z-\alpha)^{2 q}}\right] d z \wedge d \bar{z} \\
& =-\left(\frac{-1}{\tau(A)}\right)^{q} \iint_{\Omega / G} \frac{\lambda^{2-2 q}(z) \overline{\psi(z)}}{(z-\alpha)^{2 q}} d z \wedge d \bar{z} \\
& =-\iint_{\Omega / \Gamma} \varphi_{A, B}(z) \lambda^{2-2 q}(z) \overline{\psi(z)} d z \wedge d \bar{z} \\
& =i\left\langle\varphi_{A, B}, \psi\right\rangle .
\end{aligned}
$$

For a justification of the use of Stokes' theorem, see [K3, §2.4]. 
4.4. Let us assume that $\Gamma$ is finitely generated and use the notation of $\S 3.4$. We have obtained the

Corollary. The natural restrictions

$$
P H^{1}\left(\Gamma, \Pi_{2 q-2}\right) \rightarrow P H^{1}\left(\Gamma(\alpha), \Pi_{2 q-2}\right)
$$

and

$$
H^{1}\left(\Gamma, \Pi_{2 q-2}\right) \rightarrow H^{1}\left(\Gamma(\alpha), \Pi_{2 q-2}\right)
$$

are surjective provided $\alpha$ is accessible.

We note that we can replace $\Gamma(\alpha)$ by $\mathrm{P}(\alpha)$ if and only if $\alpha$ is $q$-admissible. For $\alpha$ not $q$-admissible, every cohomology class for $\Gamma$ restricts to a coboundary for $\Gamma(\alpha)$ (and thus also for $\mathrm{P}(\alpha)$ ). Example D of [K1, §7] shows that the assumption that $\alpha$ be accessible cannot be dropped.

\section{SOME FORMULAE FOR RANK 2 PARABOLIC SUBgROUPS}

5.1. Let $G$ be a subgroup of $\Gamma$. We denote by $\Theta_{G \backslash \Gamma}$ the relative Poincaré series operator mapping $q$-forms for $G$ to $q$-forms for $\Gamma$. We abbreviate $\Theta_{\{I\} \backslash \Gamma}$ by $\Theta_{\Gamma}$ and observe that

$$
\Theta_{\Gamma}=\Theta_{G \backslash \Gamma^{0}} \Theta_{G} .
$$

5.2. We consider the special case of the rank 2 parabolic group $G$ generated by

$$
A(z)=z+1, \quad B(z)=z+\tau, \quad z \in \hat{\mathbb{C}}, \operatorname{Im} \tau \neq 0 .
$$

For $k \in \mathbb{Z}^{+}$, we define

$$
g_{\tau}(z)=\frac{1}{2 \pi i} \frac{\tau(\tau-1) \cdots(\tau-k)}{(z-\tau) z(z-1) \cdots(z-k)}, \quad z \in \mathbb{C},
$$

and

$$
\Theta g_{\tau}(z)=\sum_{\gamma \in G} g_{\tau}(\gamma(z)), \quad z \in \mathbb{C} .
$$

We observe that $\Theta g_{\tau}$ is a Poincare series for the group $G$ with $q=\frac{1}{2}(k+2) \geq 2$ for $k$ even and $q=\frac{1}{2}(k+3) \geq 2$ for $k$ odd. Thus we conclude quite easily that

$$
\Theta g_{\tau}=\left(\Theta_{G}\right)\left(g_{\tau}\right)
$$

converges absolutely and uniformly on compact subsets of $\mathbb{C}$ and defines a doubly periodic function

$$
\Theta g_{\tau}(z+1)=\Theta g_{\tau}(z)=\Theta g_{\tau}(z+\tau), \quad \text { all } z \in \mathbb{C},
$$

for $G$. It is most convenient to regard $\Theta g_{\tau}$ as an automorphic form (for appropriate $q$, as above). Since $\Theta g_{\tau}$ has at most simple poles at the lattice points, it must be a constant $c(\tau)$ that depends, a priori, on $\tau$ and $k$.

Theorem. For all $\tau \in \mathbb{C}$ with $\operatorname{Im} \tau \neq 0, c(\tau)=1$. 
Proof. There exist groups $\Gamma$ (see $[\mathrm{K} 1, \S 7])$ with $G \subset \Gamma$ and $\operatorname{dim} \mathbb{A}_{q}(\Omega, \Gamma)>0$. Let us assume that $k$ is even, $k=2 q-2$ for some integer $q \geq 2$. We will obtain an alternate formula for $l_{A, B}\left(\beta^{*} \psi\right)$ with $\psi \in \mathbb{A}_{q}(\Omega, \Gamma)$. Let $F$ be the potential for $\lambda^{2-2 q} \bar{\psi}$ that vanishes at $A^{j}(0)=j, j=0,1, \ldots, 2 q-2$. Then (as usual, $l=l_{A, B}, \chi=\mathrm{pd} F$ )

$$
F(A(z))-F(z)=0, \quad \text { all } z \in \mathbb{C},
$$

and

$$
F(B(z))-F(z)=l(\chi), \quad \text { all } z \in \mathbb{C} .
$$

It follows that

$$
F(\tau)=l(\chi)
$$

But

$$
\begin{aligned}
F(\tau) & =\frac{\tau(\tau-1) \cdots(\tau-2 q+2)}{2 \pi i} \iint_{\Omega} \frac{\lambda^{2-2 q}(\zeta) \overline{\psi(\zeta)} d \zeta \wedge d \bar{\zeta}}{\zeta(\zeta-1) \cdots(\zeta-2 q+2)(\zeta-\tau)} \\
& =\iint_{\Omega / G} c(\tau) \lambda^{2-2 q}(\zeta) \overline{\psi(\zeta)} d \zeta \wedge d \bar{\zeta}
\end{aligned}
$$

Comparison with (4.3.1) yields the theorem for even $k$. For $k$ odd, we use the potential $F$ that vanishes at $\infty$ and at $0,1, \ldots, 2 q-3$.

Corollary. We have the equality

$$
\Theta g_{\tau}=h_{A}^{q}
$$
$\left[\begin{array}{ll}1 & 1 \\ 0 & 1\end{array}\right]$.

In the next section we generalize the above corollary to motions $A$ other than

Remark. Rank 2 parabolic groups figure prominently in the fundamental work of Earle and Marden [EM] on compactifications for moduli spaces. The above theorem for $q=2$ is connected to quasiconformal mappings and is clearly encountered in [EM]. It also appears in Nag's work on Teichmüller theory [N]. Nag's methods (limited to $q=2$ ) rely on the study of deformations of complex structures on tori.

5.3. Let $A$ and $B$ generate an arbitrary rank 2 parabolic group $G$. For $a \in \hat{\mathbb{C}}, a \neq \alpha=\alpha(A)=\alpha(B)$, with $B(a) \in \mathbb{C}$, define

$$
g_{A, B, a}(z)=\frac{1}{2 \pi i} \frac{1}{z-B(a)} \prod_{j=0}^{2 q-2} \frac{B(a)-A^{j}(a)}{z-A^{j}(a)}, \quad z \in \hat{\mathbb{C}} .
$$

A straightforward calculation using (1.2.1) shows that for $C \in P S L(2, \mathbb{C})$

$$
g_{C \circ A \circ C^{-1}, C \circ B \circ C^{-1}, C(a)}(C(z)) C^{\prime}(z)^{q}=C^{\prime}(B(a))^{q-1} g_{A, B, a}(z), \quad \text { all } z \in \hat{\mathbb{C}},
$$
or (equivalently)

$$
C_{q}^{*}\left(g_{C \circ A \circ C^{-1}, C \circ B \circ C^{-1}, C(a)}\right)=C^{\prime}(B(a))^{q-1} g_{A, B, a} .
$$

For $A=\left[\begin{array}{ll}1 & 1 \\ 0 & 1\end{array}\right], B=\left[\begin{array}{ll}1 & \tau \\ 0 & 1\end{array}\right]$ with $\operatorname{Im} \tau \neq 0, a=0$, we have

$$
g_{A, B, a}=g_{\tau} \text {. }
$$


Thus choosing $C \in P S L(2, \mathbb{C})$ with

$$
C(\alpha)=\infty, \quad C(a)=0, \quad C(A(a))=1,
$$

we see that

$$
C \circ A \circ C^{-1}=\left[\begin{array}{ll}
1 & 1 \\
0 & 1
\end{array}\right], \quad C \circ B \circ C^{-1}=\left[\begin{array}{ll}
1 & \tau \\
0 & 1
\end{array}\right],
$$

for some $\tau \in \mathbb{C}$ with $\operatorname{Im} \tau \neq 0$. Hence

$$
C_{q}^{*}\left(g_{C(B(a))}\right)=C^{\prime}(B(a))^{q-1} g_{A, B, a} .
$$

Assume now that $\alpha \in \mathbb{C}$. Then the above formula yields

$$
C_{q}^{*}\left(g_{\tau(B) / \tau(A)}\right)=\left(\frac{(1+\tau(B))(a-\alpha)^{2}}{-\tau(A)(a-\alpha)^{2}}\right)^{q-1} g_{A, B, a} .
$$

For $\alpha=\infty$, the corresponding formula is

$$
C_{q}^{*}\left(g_{\tau(B) / \tau(A)}\right)=\frac{1}{\tau(A)^{q-1}} g_{A, B, a} .
$$

Using the well-known relation

$$
\Theta_{G} \circ C_{q}^{*}=C_{q}^{*} \circ \Theta_{C G C^{-1}}
$$

we obtain the following

Theorem. Let $A$ and $B$ generate a rank 2 parabolic group $G$ with fixed point $\alpha$. Then

$$
\begin{aligned}
\Theta_{G}\left(g_{A, B, a}\right) & =\left(\frac{1+\tau(B)(a-\alpha)^{2}}{-\tau(A)(a-\alpha)^{2}}\right)^{1-q} h_{A}^{q} \text { for } \alpha \in \mathbb{C}, \\
& =\tau(A)^{q-1} h_{A}^{q} \text { for } \alpha=\infty
\end{aligned}
$$

Proof. We apply the Poincaré operator $\Theta_{G}$ to both sides of (5.3.1), use (5.3.3) and the Corollary to Theorem 5.2 along with (1.2.3).

Remark. Similar formulae hold for the function

$$
\frac{1}{2 \pi i} \frac{1}{z-B(a)} \frac{B(a)-\alpha}{z-\alpha} \prod_{j=0}^{2 q-3} \frac{B(a)-A^{j}(a)}{z-A^{j}(a)} .
$$

\section{REFERENCES}

[EM] C. J. Earle and A. Marden, Geometric complex coordinates for Teichmüller space (to appear).

[GK] F. P. Gardiner and I. Kra, Stability of Kleinian groups, Indiana Univ. Math. J. 21 (1972), 1037-1059.

[K1] I. Kra, On cohomology of Kleinian groups. IV. The Ahlfors-Sullivan construction of holomorphic Eichler integrals, J. Analyse Math. 43 (1983/84), 51-87.

[K2] On the vanishing of and spanning sets for Poincaré series for cusp forms, Acta Math. 153 (1984), 47-116. 
[K3] I. Kra, Cusp forms associated to loxodromic elements of Kleinian groups, Duke Math. J. 52 (1985), 587-625.

[N] S. Nag, On some involutions and retractions arising in Teichmüller spaces, J. Indian Math. Soc. 46 (1982), 1-29.

Department of Mathematics, State University of New York, Stony Brook, New York 11794-3651 